Phase transformations in a model mesenchymal tissue

This article has been downloaded from IOPscience. Please scroll down to see the full text article. 2004 Phys. Biol. 1100

(http://iopscience.iop.org/1478-3975/1/2/006)

View the table of contents for this issue, or go to the journal homepage for more

Download details:

IP Address: 128.206.162.204

The article was downloaded on 27/07/2010 at 21:50

Please note that terms and conditions apply. 


\title{
Phase transformations in a model mesenchymal tissue
}

\author{
Stuart A Newman ${ }^{1}$, Gabor Forgacs ${ }^{2}$, Bernhard Hinner ${ }^{3}$, \\ Christian W Maier ${ }^{3}$ and Erich Sackmann ${ }^{3}$ \\ ${ }^{1}$ Department of Cell Biology and Anatomy, New York Medical College, Valhalla, NY 10595, USA \\ ${ }^{2}$ Department of Physics and Biology, University of Missouri, Columbia, MO 65211, USA \\ ${ }^{3}$ Physik Department E22 (Biophysics Group), Technische Universität München, \\ D-85748 Garching, Germany \\ E-mail: newman@nymc.edu
}

Received 2 March 2004

Accepted for publication 3 June 2004

Published 25 June 2004

Online at stacks.iop.org/PhysBio/1/100

doi:10.1088/1478-3967/1/2/006

\begin{abstract}
Connective tissues, the most abundant tissue type of the mature mammalian body, consist of cells suspended in complex microenvironments known as extracellular matrices (ECMs). In the immature connective tissues (mesenchymes) encountered in developmental biology and tissue engineering applications, the ECMs contain varying amounts of randomly arranged fibers, and the physical state of the ECM changes as the fibers secreted by the cells undergo fibril and fiber assembly and organize into networks. In vitro composites consisting of assembling solutions of type I collagen, containing suspended polystyrene latex beads $(\sim 6 \mu \mathrm{m}$ in diameter) with collagen-binding surface properties, provide a simplified model for certain physical aspects of developing mesenchymes. In particular, assembly-dependent topological (i.e., connectivity) transitions within the ECM could change a tissue from one in which cell-sized particles (e.g., latex beads or cells) are mechanically unlinked to one in which the particles are part of a mechanical continuum. Any particle-induced alterations in fiber organization would imply that cells could similarly establish physically distinct microdomains within tissues. Here we show that the presence of beads above a critical number density accelerates the sol-gel transition that takes place during the assembly of collagen into a globally interconnected network of fibers. The presence of this suprathreshold number of beads also dramatically changes the viscoelastic properties of the collagen matrix, but only when the initial concentration of soluble collagen is itself above a critical value. Our studies provide a starting point for the analysis of phase transformations of more complex biomaterials including developing and healing tissues as well as tissue substitutes containing living cells.
\end{abstract}

\section{Introduction}

Early embryonic development is marked by dramatic morphogenetic processes leading to the formation of the body's plan and its specialized organs. These processes involve generation of distinct cell types, establishment of tissue boundaries, and a variety of collective motions producing cell translocations and tissue rearrangements (Gilbert 2003, Salazar-Ciudad et al 2003). All of these morphogenetic events, and those of the mature body, such as regeneration, wound healing and tumorigenesis, are driven by the interplay of specific gene regulation and generic (i.e., common to nonliving and living matter) chemical and physical processes (Newman and Comper 1990, Hutson et al 2003). Both developmental biology and tissue engineering, the production of tissue substitutes, have benefited from improved knowledge of gene regulatory processes, but the physical nature of tissues and related biomaterials remains obscure.

It has long been suggested that tissue fragments of different origin composed of adhesive and motile cells in many respects mimic the behavior of immiscible liquids (Steinberg 1978, Steinberg and Poole 1982, Armstrong 1989). 
In particular, in suspension, or on non-adhesive surfaces, various multicellular aggregates round up into a spherical shape similar to liquid droplets. Contiguously placed tissues undergo phase separation, much like oil and water, and do not exchange cells along their common border, despite the fact that the cells are mobile within each tissue mass. The molecular origin of this apparent liquid-like behavior in epithelioid tissues, in which cells make direct contact with each other, lies in differential cell adhesion, as has been demonstrated both in vitro (Steinberg and Takeichi 1994, Foty et al 1994, 1996) and in vivo (Godt and Tepass 1998, Gonzalez-Reyes and St Johnston 1998), as well as by computational means (Glazier and Graner 1993). Basically, strongly adhesive cells will maximize their contacts with other strongly adhesive cells in preference to weakly adhesive cells, thus leaving the latter to bind to one another. Although these living systems are, of course, far from thermodynamic equilibrium, the differentially adhesive cell types sort themselves out analogously to molecules of oil and water in a mixture approaching equilibrium.

Apparent phase separations occur as well during development and regeneration of mesenchymal tissues, in which cells connect to each other only through a fibrous medium known as the extracellular matrix (ECM) (e.g., Heintzelman et al (1978), Crawford and Stocum (1988), reviewed in Newman and Tomasek (1996)). A recent study (Tanaka and Tickle 2004), for example, has shown that the formation of the boundary between the wing bud and flank mesenchyme in the developing chicken embryo is due, in part, to downstream effects of the transcription factor Tbx18, which is expressed in the wing field. In the absence of direct cell-cell adhesion, a simple oil-and-water type explanation cannot apply to phase separation of mesenchymes. This phenomenon must depend, rather, on differing compositions or fiber organization of the adjoining ECMs (Forgacs et al 1989, Forgacs and Newman 1994).

Fiber organization of mesenchymal tissues can also have a dramatic effect on the behavior of the cells within them. Cells can exert tugging forces on their local ECM. If the fibers are disconnected from one another, the tugging will not be felt by distant or even nearby cells. If, however, the fibers constitute a globally connected network-either a wellstructured one like a spider web or a geodesic dome (Ingber 2003), or a randomly organized one (Forgacs 1995) — then the ECM can support mechanical communication among the cells. Percolation theory is a mathematical technique that can be used to determine whether a spanning cluster, e.g., a globally interconnected network, is present in a system containing randomly arranged, extended elements such as collagen fibers (Sahimi 1994).

To gain insight into the biophysical basis of phase separation and mechanical continuity in mesenchymal tissues, we here consider a simplified in vitro model system (Newman et al 1985, 1987), which consists of assembling collagen matrices with varying concentrations of particlesliving cells or cell-sized polystyrene latex beads. It was found (Newman et al 1985, 1987) that under appropriate conditions these materials indeed manifest immiscibleliquid-like behavior. In particular, depending on collagen concentration and number density of cells or beads, two juxtaposed preparations, a bead-containing and a bead-free collagen solution, either gradually intermix to form a single homogeneous phase, or develop a sharp moving interface, a phenomenon termed matrix-driven translocation (MDT) (Newman et al 1985). Moreover, as the interface moves, engulfment-just as observed in epithelioid tissue fragments; Steinberg and Poole (1982) — takes place: the bead-containing phase envelopes the bead-free phase. Thus, the two solutions can behave as distinct thermodynamic phases with nonzero interfacial tension (Forgacs et al 1994).

Because the two phases in the MDT experiment do not differ in molecular composition, but simply in the presence or absence of latex particles, which constitute only a small volume fraction $(<0.2 \%)$ of one of the phases, the basis for the phase separation must lie in particle-induced organizational changes of the assembling fibers. Commercial polystyrenes contain charged groups that cause them to mimic the glycosaminoglycan heparin in protein binding assays (Fougnot et al 1984, Jozefowicz and Jozefonvicz 1997). Moreover, the particles used in the MDT experiments are translocated through the ECM of the trunk neural crest pathways in living embryos (Bronner-Fraser 1982, 1984) and are conveyed into regions of developing limb bud mesenchyme rich in the ECM molecule fibronectin (Frenz et al 1989) in a fashion that depends on specific features of the particle surface and therefore behave similarly, in part, to the cells that move through the same matrices. Therefore, given the complexity of interactions between living cells and multicomponent ECMs, it seemed reasonable to study in greater detail the effect of cellsized polystyrene beads on assembling collagen matrices, as a window into some of the physical transformations potentially occurring in developing tissues.

Recently (Forgacs et al 2003) we have used light microscopy and high resolution rheometric analysis to demonstrate that solutions of type I collagen prepared with intact collagen (i.e., untreated with the enzyme pepsin, commonly used in extraction), and adjusted to physiological $\mathrm{pH}$ and ionic strength, undergo a phase transition that can be accounted for by the theory of percolation (Sahimi 1994). Specifically, over time the originally randomly distributed fibers form a spanning cluster, a globally interconnected network that extends across the entire system.

In the present work we sought to determine whether introduction of simple cell surrogates (polystyrene latex beads) into these assembling matrices causes any systematic changes in their structural and viscoelastic characteristics that may help to account for the earlier findings on their interfacial properties, and would therefore provide insight into phase separation in mesenchymal tissues. We found that the physical character, (i.e., its 'universality class' (Sahimi 1994) of the percolation phase transition undergone by the collagen matrix was unchanged by the presence of particles at all number densities studied. However, under the same conditions (bead and collagen concentrations) in which the onset of immiscibility and ensuing envelopment was observed in the MDT experiments, there were dramatic changes in the viscoelastic properties of these matrices, reflected in the 
storage and loss moduli (related, respectively, to elasticity and viscosity). Our results suggest that mechanical continuity can be established in ECMs of mesenchymal tissues by a percolation phase transition, and indicate that resident particles with collagen-binding properties (cells as well as beads) can induce significant changes in matrix viscoelasticity.

\section{Materials and methods}

\subsection{Sample preparation}

Type I collagen was extracted from the tails of young adult rats with $3 \%$ acetic acid, as described previously (Elsdale and Bard 1972, Newman et al 1985, Forgacs et al 2003), and exchanged into Ham's F-12, the tissue culture medium in which the MDT effect was first characterized for living cells (Newman et al 1985). The acetic acid extract was adjusted to a protein concentration of 3 to $5 \mathrm{mg} \mathrm{ml}^{-1}$ and dialyzed for 2 days against two changes of 1/10 strength Ham's F-12 medium without bicarbonate (Gibco-Life Technologies, Grand Island, NY). This solution was brought to full strength Ham's F12 in the following fashion: to $0.7 \mathrm{ml}$ of dialyzed collagen solution on ice was added in succession, with rapid mixing, $0.1 \mathrm{ml} 9.3 \times$ Ham's F-12, $0.1 \mathrm{ml}$ distilled water, and $0.1 \mathrm{ml}$ sodium bicarbonate $\left(11.76 \mathrm{mg} \mathrm{ml}^{-1}\right)$. This, in turn, was rapidly mixed with $1 \mathrm{ml}$ of complete Ham's F-12. In order to prepare bead-containing solutions, $6 \mu \mathrm{m}$ polystyrene latex beads (Polysciences, Warrington, PA) were added at twice the desired final concentration to the $1 \mathrm{ml}$ of complete Ham's F-12 medium used in the final step. The beads were washed extensively in distilled water prior to adding them to the medium, to remove traces of surfactant included by the manufacturer. To ensure monodispersity of the beads, the F-12 bead suspensions were subjected to sonic disruption for 5 min (Bandelin Sonorex RK 100, Bandelin Electronic, Berlin, Germany) before mixing them with equal volumes of soluble collagen. The collagen solutions were kept on ice for controlled amounts of time until being transferred to experimental chambers as described below. As with the beadfree collagen solutions described by Forgacs et al (2003), experimental values of the viscoelastic parameters obtained on the same day were highly reproducible, but differed from determinations on other days in a manner that increased with the time difference between the preparation time of the collagen stock solution (stored at $4{ }^{\circ} \mathrm{C}$ ) and the measurements. Comparisons shown on the same graph therefore refer always to measurements made on the same day.

\subsection{Microstructural studies of collagen gels}

Samples for microstructural studies were prepared as follows. The collagen solution was injected into a sample chamber, thermostated at $12{ }^{\circ} \mathrm{C}$, consisting of two horizontal, parallel coverslips separated by a distance of $2 \mathrm{~mm}$. Phase contrast images were taken using an Axiovert 100 inverted microscope (Carl Zeiss, Jena, Germany) equipped with an Achrostigmat $20 \times / 0.3$ LD Ph1 and a Plan-Neofluar 100×/1.3 Oil Ph3 lens. Micrographs were recorded with a CCD camera and stored on SVHS video tape. For further analysis, images were first digitized using a Macintosh 9500 computer (Apple Computer, Cupertino, CA) equipped with a Scion LG3 frame grabber card (Scion Corporation, MD) and subsequently analyzed and processed using NIH Image software (NIH, Bethesda, MD). In order to reduce noise, first the final image was obtained by averaging over 20 consecutive images. Subsequently, a ranking filter (median mode) was applied. Finally, a pseudoshadowing filter (part of the NIH Image software) was used to improve the visibility of details.

\subsection{Measurement of viscoelastic properties}

Measurements using a rotating disc microrheometer were performed as described by Forgacs et al (2003). Details on the rotating disc rheometer have been given by Müller et al (1991). Briefly, the rheometer consists of a cylindrical cuvette with an inner diameter of $15 \mathrm{~mm}$ and a volume of about $1.5 \mathrm{ml}$. The base of the cuvette is mounted in an aluminum thermostated holder. A silanized glass disc with a diameter of $8 \mathrm{~mm}$ is placed on the surface of the sample. Mounted on top of the disc are a magnet and deflection mirror with dimensions of $0.5 \times 0.5 \times 1.0 \mathrm{~mm}^{3}$ and $1.5 \times 2.0 \mathrm{~mm}^{2}$, respectively. The cuvette is surrounded by two perpendicularly oriented pairs of magnetic coils. One of these pairs provides a static magnetic field to fix the orientation of the disc. The other pair of coils is driven by a sinusoidally varying voltage of frequency $\omega$ to apply an oscillatory shear stress to the sample by means of the silanized glass disc. To improve its sensitivity, the rheometer is shielded from external magnetic fields (e.g., the magnetic field of the earth) by a cage of high magnetic permeability metal (Vakuumschmelze Hanau, Hanau, Germany). The rotational amplitude of the disc is analyzed as follows: the beam of a $\mathrm{He}-\mathrm{Ne}$ laser is deflected by the mirror on top of the glass disc, and its position is detected by a position-sensitive photodiode; the resulting signal is amplified and evaluated by a computer.

The viscoelastic moduli, storage modulus $G^{\prime}(\omega)$ (related to the shear elastic modulus in the $\omega \rightarrow 0$ limit) and loss modulus $G^{\prime \prime}(\omega)$ (or the dynamic viscosity $\eta(\omega)=$ $\left.G^{\prime \prime}(\omega) / \omega\right)$ were determined from the amplitude of the glass disc oscillation and the phase shift angle $\varphi$ between this oscillation and the applied oscillatory torque (Fung 1993, Müller et al 1991). Measurements were performed as follows: a $400 \mu \mathrm{l}$ aliquot of collagen solution (prepared on ice) was transferred into the measuring chamber, this instant defining the zero time of the kinetic measurements. Four minutes after transfer the measurement was started at a fixed frequency $\omega$. A value of $G^{\prime}(\omega)$ and $G^{\prime \prime}(\omega)$ was measured every $45 \mathrm{~s}$ to determine their time evolution, $G_{\omega}^{\prime}(t)$ and $G_{\omega}^{\prime \prime}(t)$. For each studied frequency, a new sample was prepared and the same protocol was applied. In this way it was also possible to determine the frequency-dependent impedance spectra, $G_{t}^{\prime}(\omega)$ and $G_{t}^{\prime \prime}(\omega)$, for consecutive times $t$. If required, the viscoelastic moduli at intermediate times were obtained by interpolating the measured data points (by means of cubic spline functions).

\subsection{Percolation}

Data analysis was performed using percolation theory (De Gennes 1988, Stauffer and Aharony 1992, Sahimi 1994). 
(a)
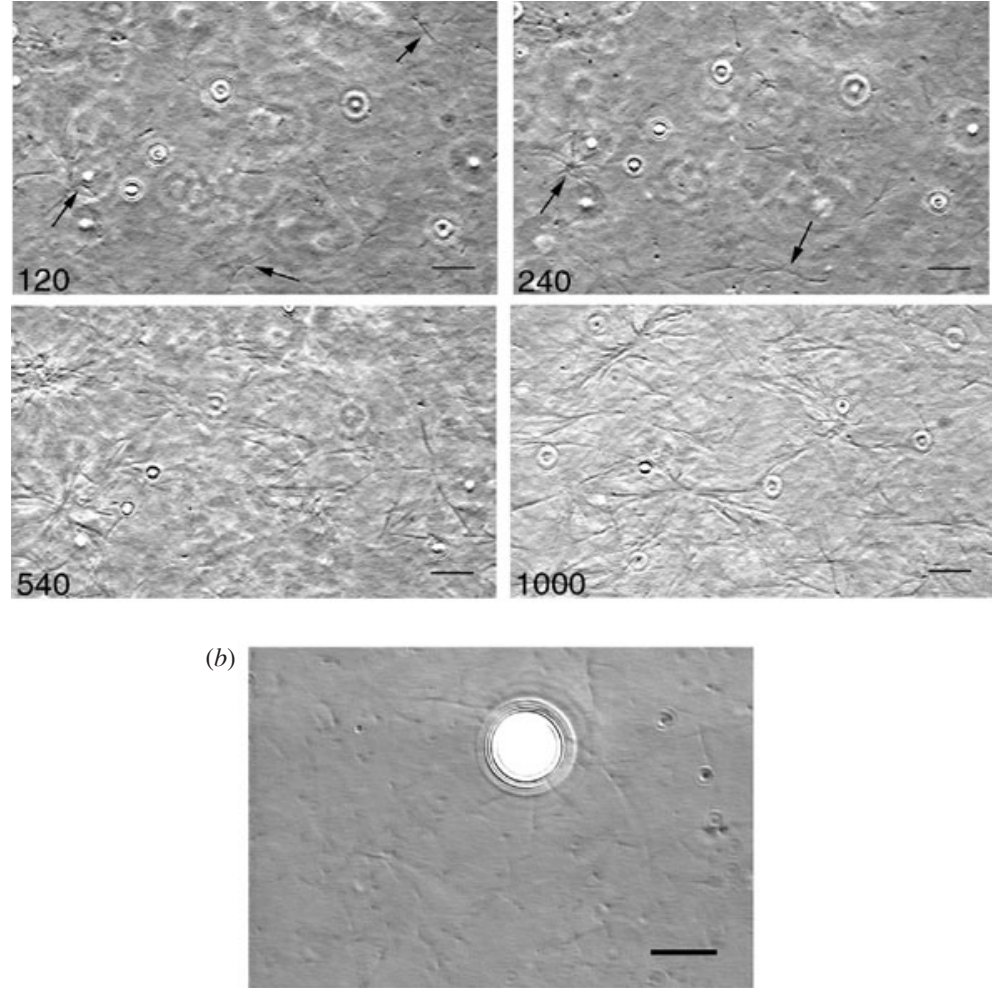

Figure 1. (a) Phase contrast image of bead-containing collagen solutions (collagen concentration $c=1.7 \mathrm{mg} \mathrm{ml}{ }^{-1}$, bead concentration $c_{b}=3 \times 10^{6} \mathrm{ml}^{-1}$ ) at $T=12^{\circ} \mathrm{C}$ viewed with a $20 \times$ objective. The first three images have been taken at the same location, with only a slight change of the focal plane. The fourth image shows the network at another site. At $120 \mathrm{~s}$ a few well-developed fibers can be seen (denoted by arrows). Also the contour of a branched structure emerges at the left of the image (indicated by an arrow). Two minutes later much longer fibers can be seen (see arrow at the lower edge of the image), whereas the branched structure is much more pronounced. By $540 \mathrm{~s}$ the network has percolated. The last image shows the extensive cross-linking of the fibers. Scale bar $20 \mu \mathrm{m}$. $(b)$ Phase contrast micrograph of the same collagen solution as in $(a)$ viewed with a $100 \times$ objective. Note the radial arrangement of collagen fibers in the vicinity of the latex particle. Scale bar $5 \mu \mathrm{m}$.

This theory stipulates that gelation in a filamentous macromolecular system is due to the gradual interconnection of growing fibers. Below the transition only small isolated clusters of interconnected fibers exist. However, at a threshold value of connections, the isolated clusters become interconnected and form a continuous network, which defines the sol-gel transition. Percolation theory predicts power law behavior for the macroscopic physical properties such as the elastic modulus or viscosity in the vicinity of the gelation point. In the present work we use the prediction of this theory for the behavior of the $\omega$ dependent complex viscoelastic modulus at the transition (Durand et al 1987)

$$
G^{*}(\omega)=G^{\prime}(\omega)+\mathrm{i} G^{\prime \prime}(\omega) \cong \omega^{\Delta} \exp (\mathrm{i} \pi \Delta / 2) .
$$

Equation (1) implies identical scaling laws for $G^{\prime}(\omega)$ and $G^{\prime \prime}(\omega)$ at the gel point, $G^{\prime}(\omega) \propto G^{\prime \prime}(\omega) \propto \omega^{\Delta}$. (Equation (1) is the customary way to write a complex quantity with real and imaginary parts with $\mathrm{i}=\sqrt{-1}$.) It also follows from equation (1) that at the gel point the critical loss angle $\delta_{\mathrm{c}}(\delta$ is defined as $\tan \delta=G^{\prime \prime} / G^{\prime}$ ) is related to the exponent $\Delta$ by

$$
\delta_{\mathrm{c}}=\frac{\pi}{2} \Delta .
$$

\section{Results and discussion}

\subsection{Phase contrast microscopy}

Gelation of collagen in the presence of latex beads exhibited no major differences from that in the absence of beads when viewed by phase contrast microscopy using a $20 \times$ objective (compare figure 1(a) with figure 1 in Forgacs et al (2003)). (The studies on bead-free collagen reported in Forgacs et al (2003) were performed contemporaneously with the measurements described here, and therefore serve as appropriate reference data for the present studies.) However, use of a $100 \times$ objective suggests that beads affect the structure of the assembling network by locally rearranging or nucleating collagen fibers (figure $1(b)$ ).

\subsection{Rheometry}

The viscoelastic parameters $G_{\omega}^{\prime}(t)$ and $G_{\omega}^{\prime \prime}(t)$ of the beadcontaining solutions were measured as functions of time for five different frequencies (figure 2), using a collagen concentration of $1.7 \mathrm{mg} \mathrm{ml}^{-1}$ and a bead number density of $3.0 \times 10^{6} \mathrm{ml}^{-1}$. The general features of these results are similar to the bead-free case (Forgacs et al 2003). 

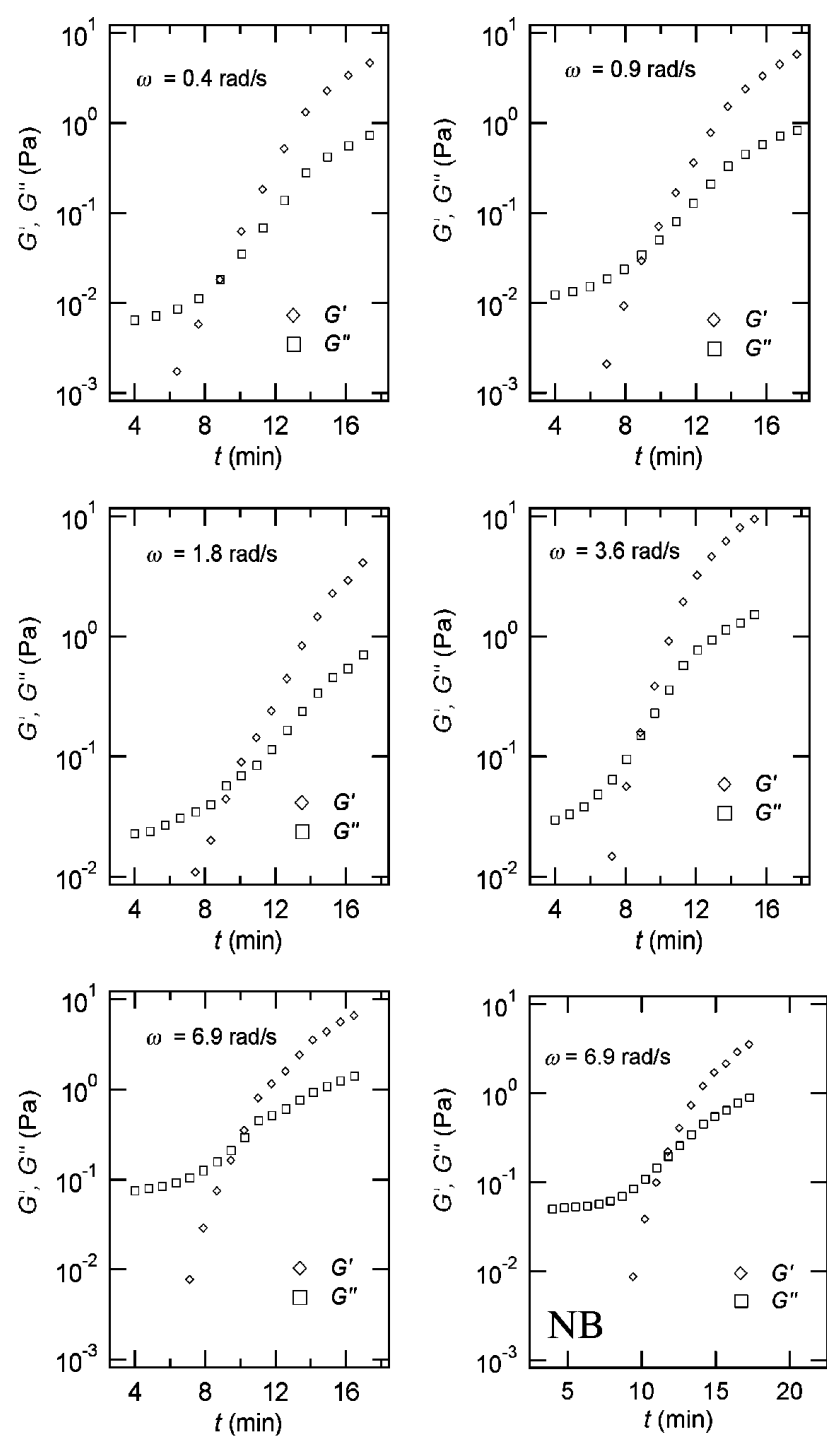

Figure 2. The first five graphs show the time dependence of the viscoelastic moduli $G_{\omega}^{\prime}(t)$ and $G_{\omega}^{\prime \prime}(t)$ of the same collagen solution as in figure 1 for five different frequencies. The graph with label NB shows analogous results for a $1.7 \mathrm{mg} \mathrm{ml}^{-1}$ collagen solution with no beads. Comparison of the two graphs in the last line indicates that beads speed up gelation (see text). The comparison is shown here only for one frequency, but similar trend can be observed for all the frequencies used in the experiments (cf with figure 2 in Forgacs et al (2003)).

However, comparison with contemporaneously prepared beadfree samples reveals that the latex particles speed up the gelation process. As shown in figure 2, for all frequencies the first nonvanishing values of $G^{\prime}$ appear at around $7 \mathrm{~min}$ (9 $\mathrm{min}$ in the bead-free case), and the intersection of $G^{\prime}$ and $G^{\prime \prime}$ now takes place at around $9 \mathrm{~min}$ (>10 $\mathrm{min}$ for the bead-free case; Forgacs et al (2003)).

The viscoelastic impedance spectra, $G_{t}^{\prime}(\omega)$ and $G_{t}^{\prime \prime}(\omega)$, at different times after sample preparation are shown in figure 3 . Up to $240 \mathrm{~s}$ the sample behaved as a Newtonian liquid, i.e., the loss modulus scales linearly with frequency (implying a frequency-independent viscosity) and there was no detectable
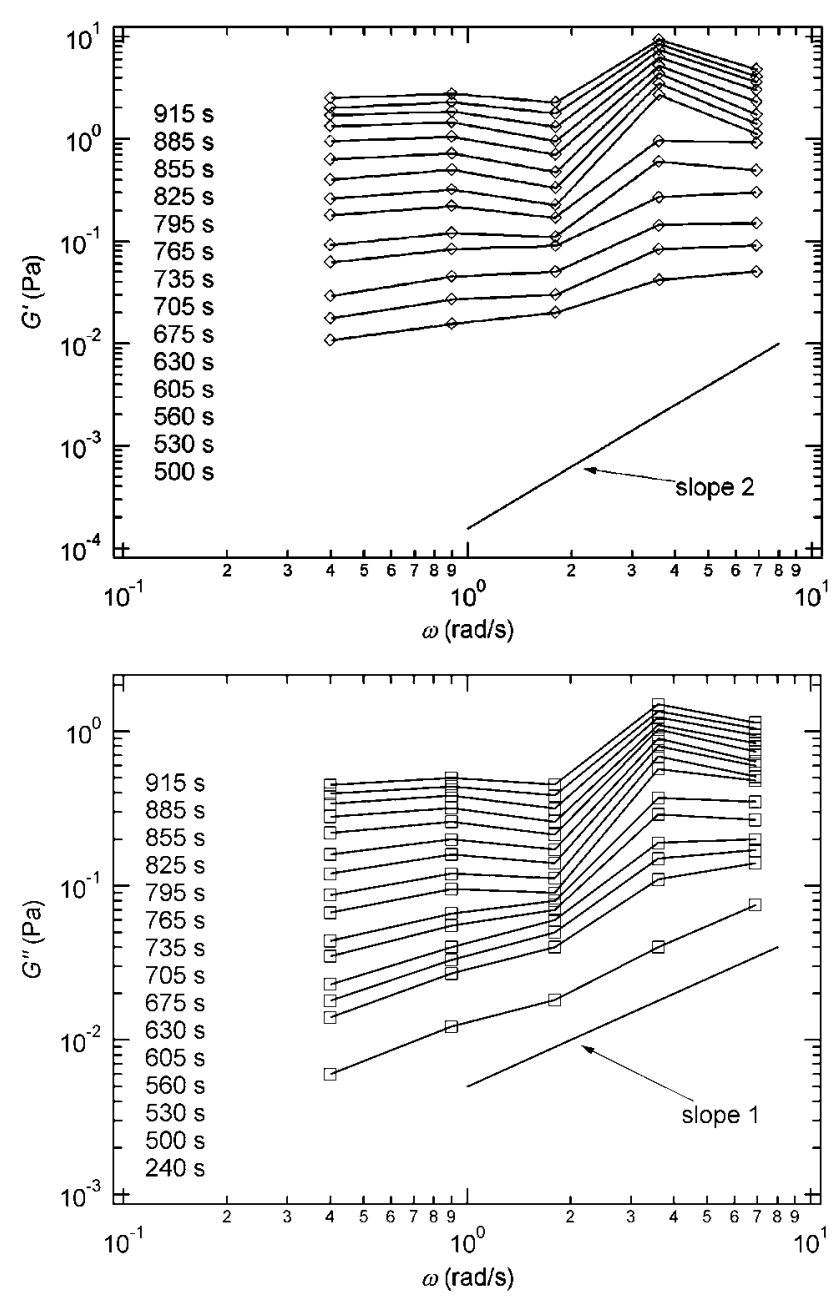

Figure 3. Viscoelastic impedance spectra $G_{t}^{\prime}(\omega)$ and $G_{t}^{\prime \prime}(\omega)$ of the same collagen solution as in figure 1 . The straight lines at the bottom represent the scaling laws of $G_{t}^{\prime}(\omega)$ and $G_{t}^{\prime \prime}(\omega)$ for the Maxwell model (Fung 1993). Upper panel: storage modulus. Lower panel: loss modulus.

storage modulus. However, at later times the fluid behavior became more complex. A nonlinear least-square fit to the data at $500 \mathrm{~s}$ indicates that at this time $G_{t}^{\prime}(\omega)$ and $G_{t}^{\prime \prime}(\omega)$ are both growing as $\omega^{\Delta}$ with $\Delta \approx 0.7$ (figure 4). This defines the gel point, or gelation transition, in processes governed by percolation; see section 2 (Forgacs et al 2003, Durand et al 1987, De Gennes 1988).

Earlier work indicated that the presence of a critical number density of beads (or cells) induced changes in the physical properties of the assembling collagen matrix such that it became immiscible with the bead-free matrix (Newman et al 1985, Forgacs et al 1989). We therefore studied the effect of varying bead concentration on the viscoelastic moduli. Our results for $\omega=3.6 \mathrm{rad} \mathrm{s}^{-1}$ are summarized in figure 5. We note that the values of $G^{\prime}$ and $G^{\prime \prime}$ for the bead concentration $0.3 \times 10^{6} \mathrm{ml}^{-1}$ are similar to those for collagen containing no beads, whereas these values differ at all time points from those at 1.2 and $3.0 \times 10^{6} \mathrm{ml}^{-1}$, which are nonetheless similar to one another. These observations suggest that at a threshold number 
Phase transformations in a model mesenchymal tissue

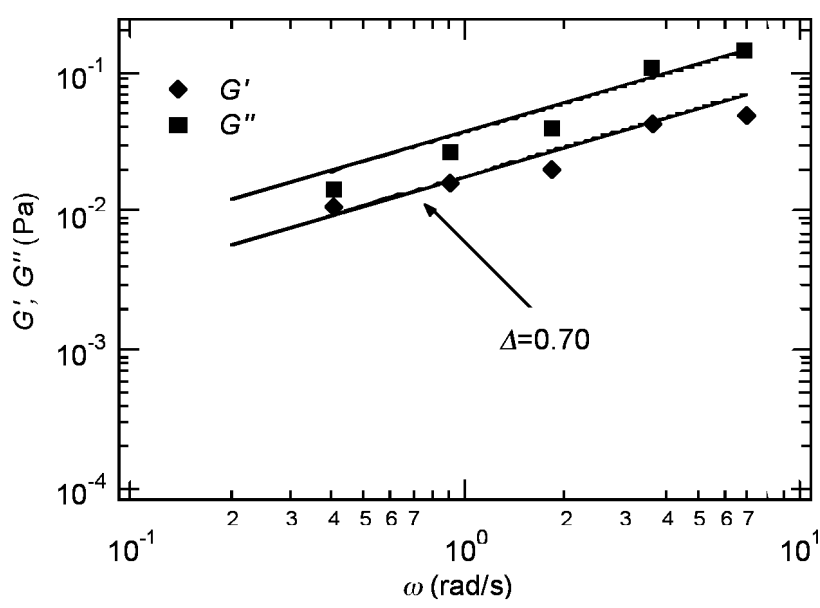

Figure 4. Log-log plot of the viscoelastic moduli as a function of frequency in the vicinity of the gel point at $t=500 \mathrm{~s}$. $G_{t}^{\prime}(\omega)$ and $G_{t}^{\prime \prime}(\omega)$ obey identical scaling laws with an exponent $\Delta \propto 0.70$.
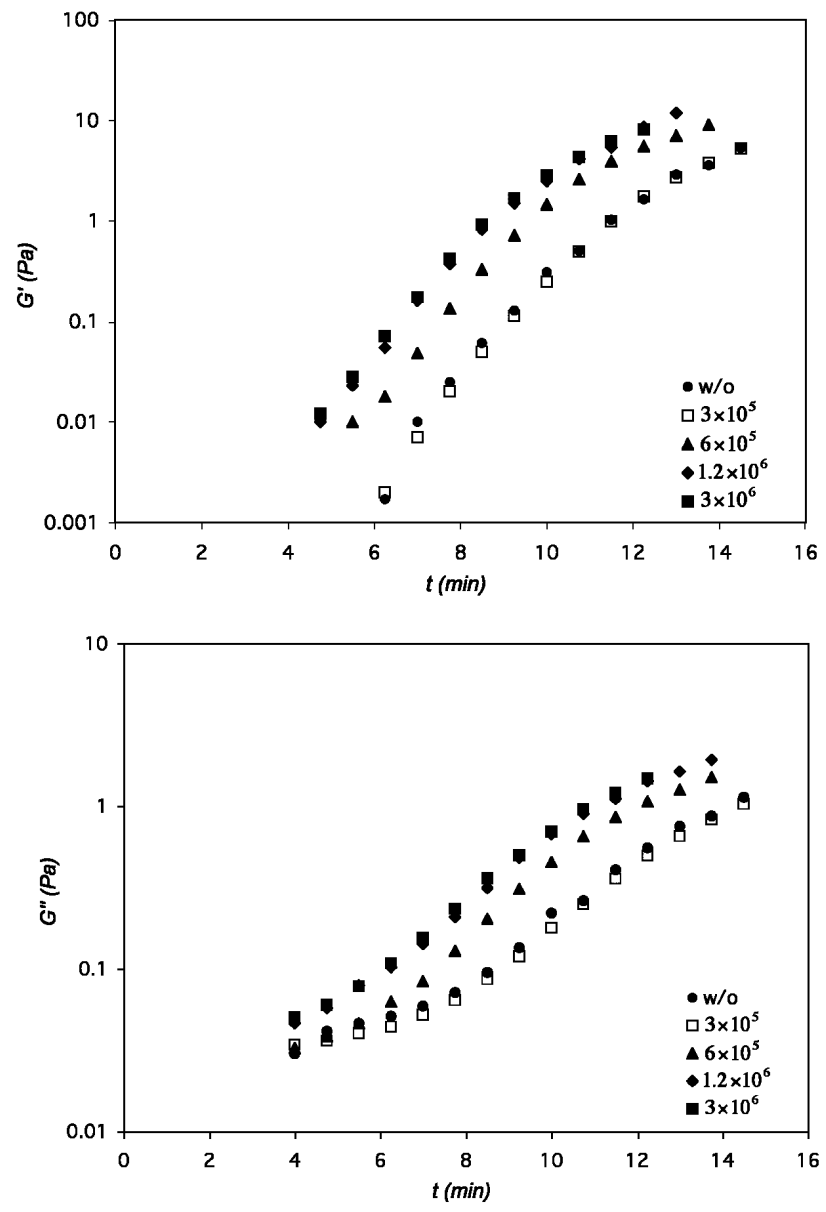

Figure 5. Bead concentration dependence of the viscoelastic moduli $G_{\omega}^{\prime}(t)$ (upper panel) and $G_{\omega}^{\prime \prime}(t)$ (lower panel) $\left(c=1.7 \mathrm{mg} \mathrm{ml}^{-1}\right.$, $\left.T=12^{\circ} \mathrm{C}, \omega=3.6 \mathrm{rad} \mathrm{s}^{-1}\right)$. Numbers in the figures indicate the number of beads in $1 \mathrm{ml}$ of the suspensions with 'w/o' denoting the bead-free solution.

density of beads the assembling collagen matrix becomes rheologically distinct from its unpopulated counterpart, and
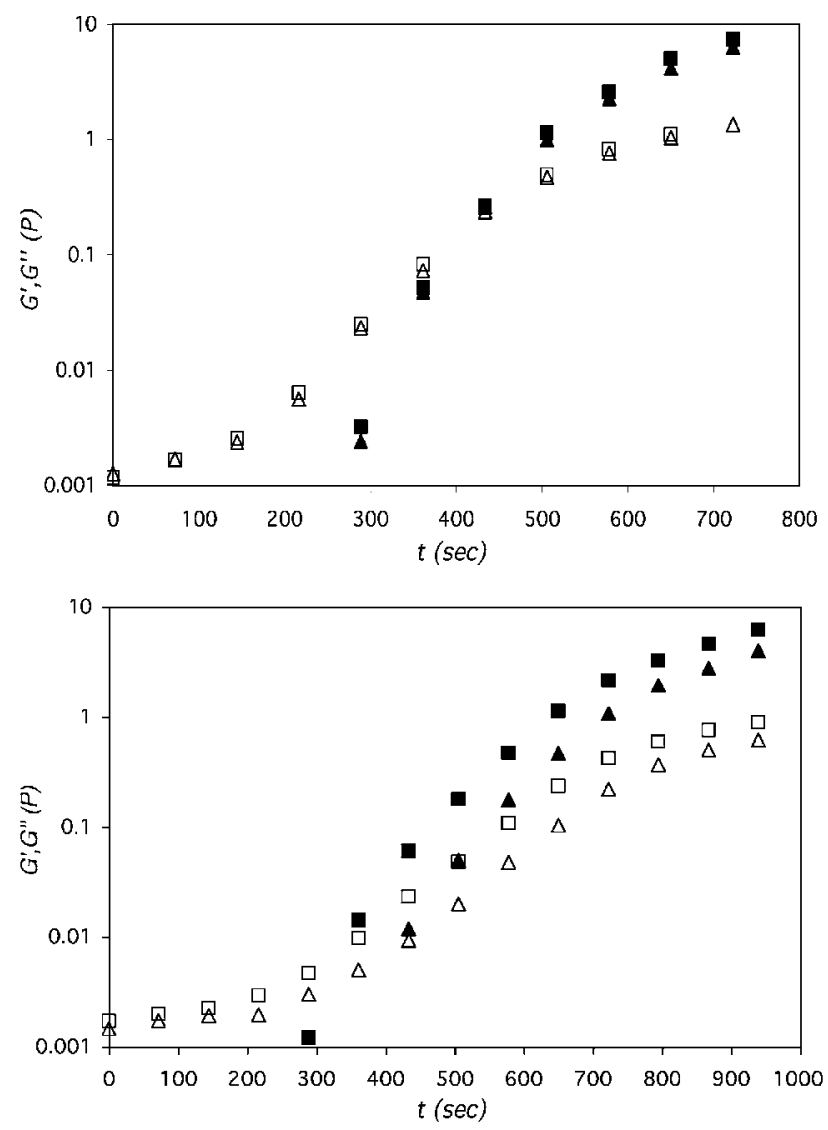

Figure 6. Collagen concentration dependence of the viscoelastic moduli $\left(T=12{ }^{\circ} \mathrm{C}, \omega=0.2 \mathrm{rad} \mathrm{s}^{-1}, c_{b}=3 \times 10^{6} \mathrm{ml}^{-1}\right)$. Upper panel: $c=1.4 \mathrm{mg} \mathrm{ml}^{-1}$. Lower panel: $c=1.7 \mathrm{mg} \mathrm{ml}^{-1}$. Closed and open symbols denote respectively $G_{\omega}^{\prime}(t)$ and $G_{\omega}^{\prime \prime}(t)$. Triangles and squares indicate samples with no beads and a supercritical number density of beads $\left(3.0 \times 10^{6} \mathrm{ml}^{-1}\right)$, respectively.

that once this transition occurs additional beads have little effect. Experiments performed at other frequencies gave similar results (not shown).

Our earlier studies using the MDT assay indicated that the abrupt change in physical properties induced by a critical number density of particles occurred only for values of collagen concentration, $c>1.6 \mathrm{mg} \mathrm{ml}^{-1}$ (Newman et al 1985 , Forgacs et al 1989, 1994). We therefore studied the effect of collagen concentration on the viscoelastic parameters in the absence of beads and in the presence of a supracritical bead concentration $\left(3.0 \times 10^{6} \mathrm{ml}^{-1}\right)$ (figure 6). Our results show the differences between the viscoelastic characteristics of the bead-containing and bead-free matrices are much more pronounced at $c=1.7 \mathrm{mg} \mathrm{ml}^{-1}$ than at $c=1.4 \mathrm{mg} \mathrm{ml}^{-1}$, implying that at the lower concentration the presence of beads has little effect on the values of $G^{\prime}$ and $G^{\prime \prime}$.

It was possible to consistently interpret all the experimental results on bead-free assembling type I collagen matrices at protein concentrations greater than $1.6 \mathrm{mg} \mathrm{ml}^{-1}$ in terms of a gelation-percolation transition (Forgacs et al 2003), at which the fibers in these matrices become globally interconnected. Our results with bead-containing matrices indicate that the percolation model of gelation applies also 
to the bead-containing case (figure 4 ). At $t=500 \mathrm{~s}$ the two viscoelastic moduli show the characteristic power law behavior predicted by percolation theory, $G_{t}^{\prime}(\omega) \propto G_{t}^{\prime \prime}(\omega) \propto \omega^{\Delta}$, with $\Delta \approx 0.7$ (Durand et al 1987, Forgacs et al 2003). Similar results were obtained for the bead-free case: the storage and loss moduli scale with the same exponent $\Delta \approx 0.7$ ) but the gelation transition occurs at a later time, namely $605 \mathrm{~s}$ (Forgacs et al 2003).

The above results imply that the presence of beads does not alter the universal (Sahimi 1994) features of the transition (i.e., the value of the exponent $\Delta$ ). Specifically, the fact that the unpopulated and populated matrices undergo a percolation transition with the same universal properties, but can have quite distinct viscoelastic properties during the fibril assembly process (figures 5 and 6 ), shows that such a transition is generic to a wide range of composite materials based on assembling type I collagen matrices, a category that includes developing connective tissues.

\section{Conclusions and outlook}

Earlier experiments using the MDT assay (Newman et al 1985, Forgacs et al 1989) provided evidence that particle (bead or cell)-containing matrices could phase-separate from particle-free matrices in a certain range of bead and collagen concentrations. These concentrations were similar to those that led to measurable differences in values of physical parameters of the matrices, i.e., interfacial tensions (Forgacs et al 1994) and rates of fibrillogenesis (Newman et al 1997). With the present work we have extended our knowledge of the properties of these matrices by considering their structural organization and rheological properties. Specifically, we found differences in both the structural organization and the viscoelastic behavior of bead-containing and bead-free collagen matrices. These differences occur within the same bead and collagen concentration ranges as those leading to the apparent phase separation between adjoining matrix regions and their subsequent mutual envelopment in the MDT experiments (Newman et al 1985, Forgacs et al 1989) and the same range of collagen concentration in which seeded connective tissue cells can contract a formed gel (Nakagawa et al 1989, Sawhney and Howard 2002).

For collagen concentrations greater than $1.6 \mathrm{mg} \mathrm{ml}^{-1}$ and bead concentrations of the order of $10^{6} \mathrm{ml}^{-1}$, nonzero values of the storage modulus (reflecting tissue elasticity), appear at earlier times, and the values of the loss modulus (reflecting tissue viscosity) are larger, than in the absence of beads. Mature connective tissues can have collagen concentrations as high as $50 \mathrm{mg} \mathrm{ml}^{-1}$ (Karim et al 1995) and $1 \mathrm{~cm}^{3}$ of an epithelioid tissue contains about $10^{9}$ cells. These are upper limits; mesenchymal tissues, particularly at early stages of development, will have values of collagen concentration and cell number density that are at least an order of magnitude lower.

The alterations of matrix organization and mechanical properties that occur during fibrillogenesis with the addition of particles-cells, or cell-sized beads-are of particular interest with respect to mesenchymal tissue developmental mechanisms and potential tissue engineering applications. Translocating mesenchymal cells in embryos, despite not being in direct contact with their neighbors, typically move as if they were part of mechanically uniform media (Nakatsuji et al 1986, Newgreen 1989). As mentioned above, like epithelioid tissues (Steinberg 1978, Steinberg and Poole 1982), mesenchymal tissues may undergo apparent phase separation, as in the formation of the limb bud primordia along the flank of tetrapod embryos (Heintzelman et al 1978, Tanaka and Tickle 2004) and during limb regeneration (Crawford and Stocum 1988). In addition, mechanical continuity is an increasingly recognized mode of cell-cell communication (Ingber 2003), but the specific requirements on the organization of a fibrous matrix necessary to achieve such continuity are not well understood. The results reported here suggest the basis for some of these behaviors and properties, and indicate that the matrix does not have to have a welldefined architecture (Ingber 2003) to serve as a tissue-wide communication medium.

The polystyrene latex particles used in our studies are roughly the size of cells, and contain molecular moieties on their surfaces that functionally mimic heparin and the heparan sulfate proteoglycans found on cell surfaces (Fougnot et al 1984, Jozefowicz and Jozefonvicz 1997). As noted in the introduction, these beads are translocated through ECM-rich spaces of neural crest pathways of living embryos and limb mesenchyme in vitro. In both these cases they behave like similar beads coated with heparin (Bronner-Fraser 1982, 1984, Frenz et al 1989). While MDT occurs with uncoated or heparin-coated $6 \mu \mathrm{m}$ beads, it fails to occur with $0.2 \mu \mathrm{m}$ beads, or $6 \mu \mathrm{m}$ beads coated with dextran sulfate, which has similar charge and composition to heparin, but a different molecular configuration (Newman et al 1985). Heparin binds to assembling type I collagen and has a dramatic effect on fibril and fiber architecture (McPherson et al 1988). Thus, the latex beads may, in certain respects, mimic cells, allowing the bead-containing matrices to serve as a simplified model for mesenchymal tissues.

It must also be recognized that there are practical problems standing in the way of performing experiments like those described here with living cells, as preferable as this might be. Our rheological measurements were performed at $12{ }^{\circ} \mathrm{C}$ to extend the duration of fibrillogenesis to several minutes. At physiological temperatures our preparations gel in less than 1 min. Of course active cellular processes would be impaired at our assay temperature, and it is not clear that even passive surface properties would be normal. In living tissues collagen fibrillogenesis occurs in a complex environment containing glycoproteins, proteoglycans and the glycosaminoglycan hyaluronan (Newman and Tomasek 1996), some of which may serve to slow down this process. In principle, microrheological studies could be performed in such composite matrices at typical body temperatures in the presence of living cells, but physical models for the timedependent behavior of these materials would be much more complex than that used here.

The similarity between collagen assembly in vivo and that in the model mesenchymes we have analyzed is not exact. 
In living tissues, fibril subunits are preformed intracellularly and assemble into fibrils and fibers extracellularly. Our acetic acid-extracted collagen similarly consists of partially assembled fibrils, but their size distribution is likely to differ from those in the extracellular space. Nonetheless, in both the in vivo and in vitro cases the density of fibers is initially low and fibril subunits and fibrils associate laterally and end-to-end, producing fibers with similar ultrastructural appearance (Elsdale and Bard 1972). It is therefore reasonable to hypothesize that collagen matrices assembling in vivo may undergo physical changes like those described in our in vitro system.

In contrast to mature connective tissues, in which type I collagen fibers exhibit extensive organization (Bouligand 1972, Gaill et al 1991, Cleary 1996, Veis 1996, Giraud-Guille 1996, Linsenmayer et al 1998), immature mesenchymal tissues exhibit no obvious organization of fibrils or fibers (Thorogood and Hinchliffe 1975, Duband and Thierry 1987). In the absence of detailed physical knowledge it would be reasonable to characterize such composite materials as 'amorphous'. However, the phenomenon of percolation (Sahimi 1994) shows that a network that meets certain criteria, even if constructed from randomly arranged elements, can develop specific topological properties not shared by typical amorphous materials.

Networks, even if randomly constructed, can serve as communication media (Barabasi 2002). In tissues, these communication networks can operate within (Forgacs 1995, Shafrir et al 2000, Shafrir and Forgacs 2002) and between cells. Cells can attach to (Gullberg and Lundgren-Ackerlund 2002, Miranti and Brugge 2002) and exert force (Roy et al 1999, Zahalak et al 2000, Freyman et al 2001) on their microenvironments. If the microenvironment is mechanically continuous the forces can be transmitted over macroscopic distances.

The bases for the surface tension differences between assembling collagen matrices populated by biologically realistic numbers of cell-sized particles (Forgacs et al 1994) and their apparent phase separation in the MDT experiments (Newman et al 1985, 1987, Forgacs et al 1989) remain elusive. However, the studies reported here and in Forgacs et al (2003) provide insight into phase transformations affecting these materials and physical differences between them, and support models that suggest that it is the differences in structural organization of these matrices that eventually give rise to their phase separation (Forgacs et al 1989, 1991, Newman et al 1997, Newman 1998). A more complete understanding of the phase separation phenomenon is likely to provide insight into mesenchymal morphogenesis and principles for the design of tissue substitutes.

\section{Acknowledgments}

We thank M A Dichtl, K Kroy and R Merkel for valuable suggestions and stimulating discussions and $\mathrm{V}$ Parsegian for advice on the presentation. This work was supported by the Deutsche Forschungsgemeinschaft (Sa. 246/22-4, a group grant SFB 266), the Fonds der Chemischen Industrie, and grants from the National Science Foundation to GF (IBN-97100010) and SAN (IBN-9603838) and NASA to GF (NAG2-1611).

\section{Glossary}

Epithelioid. A category of tissue in which cells make direct contact with their neighbors via membrane bound adhesion proteins.

Gel point. The instant when a solution containing polymerizing monomers develops a spanning network of interconnected polymers with apparent elastic properties; at the gel point a special percolation transition (i.e., sol-gel transition) takes place.

Loss angle ( $\delta)$. A quantity constructed from the loss and storage moduli, $\tan \delta=G^{\prime \prime} / G^{\prime}$, whose absolute value (between $\pi / 2$ and 0 ) gives a measure of how liquid (sol-like) or elastic (gel-like) is the viscoelastic material.

Loss modulus $\left(G^{\prime \prime}\right)$. One of the physical parameters characterizing a viscoelastic material, its ability to dissipate energy.

Matrix-driven translocation. A phenomenon identified in extracellular matrices constructed in vitro, in which a particle (cell, bead)-containing collagenous matrix phase separates from and partially engulfs a particle-free matrix of similar composition.

Mesenchyme. A category of tissue, common to the early embryo and early stages of organogenesis, in which cells are loosely suspended in a dilute extracellular matrix.

Percolation theory/transition. A mathematical representation of a (second-order) phase transition in the connectivity properties of systems made up of randomly or irregularly arranged subunits (e.g., filaments, monomers, metallic islands in an insulating matrix, etc.): below the transition, the system contains isolated clusters of the subunits, whereas above the transition, arrived at by increasing the number of subunits, an interconnected network of the subunits forms which spans the entire system.

Spanning cluster. The interconnected cluster of subunits that forms at the percolation transition and extends over the entire system.

Storage modulus $\left(G^{\prime}\right)$. One of the physical parameters characterizing a viscoelastic material, its capacity to store elastic energy.

Universality class. The set of those physical systems undergoing a (second-order) phase transition, which-despite differences in their molecular interactions on the microscopic scale, insofar as the functional dependence of their characteristic physical properties on the deviation from the transition point is concerned-manifest identical behavior. 


\section{References}

Armstrong P B 1989 Cell sorting out: the self-assembly of tissues in vitro Crit. Rev. Biochem. Mol. Biol. 24 119-49

Barabasi A-L 2002 Linked: The New Science of Networks (Cambridge, MA: Perseus)

Bouligand Y 1972 Twisted fibrous arrangements in biological materials and cholesteric mesophases Tissue Cell 4 189-217

Bronner-Fraser M 1982 Distribution of latex beads and retinal pigment epithelial cells along the ventral neural crest pathways Dev. Biol. 91 50-63

Bronner-Fraser M 1984 Latex beads as probes of a neural crest pathway: effects of laminin, collagen, and surface charge on bead translocation J. Cell Biol. 98 1947-60

Cleary E G 1996 Skin Extracellular Matrix (Tissue Function vol 1) ed W D Comper (Amsterdam: Harwood Academic) pp 77-109

Crawford K and Stocum D L 1988 Retinoic acid coordinately proximalizes regenerate pattern and blastema differential affinity in axolotl limbs Development 102 687-98

De Gennes P G 1988 Scaling Concepts in Polymer Physics (Ithaca, NY: Cornell University Press)

Duband J L and Thiery J P 1987 Distribution of laminin and collagens during avian neural crest development Development 101 461-78

Durand D, Delsanti M, Adam M and Luck J M 1987 Frequency dependence of viscoelastic properties of branched polymers near gelation threshold Europhys. Lett. 3 297-301

Elsdale T and Bard J 1972 Collagen substrata for studies on cell behavior J. Cell Biol. 54 626-37

Forgacs G 1995 On the possible role of cytoskeletal filamentous networks in intracellular signaling: an approach based on percolation J. Cell Sci. 108 2131-43

Forgacs G, Jaikaria N S, Frisch H L and Newman S A 1989 Wetting, percolation and morphogenesis in a model tissue system J. Theor. Biol. 140 417-30

Forgacs G, Newman S A, Hinner B, Maier C W and Sackmann E 2003 Assembly of collagen matrices as a phase transition revealed by structural and rheologic studies Biophys. J. 84 $1272-80$

Forgacs G, Newman S A, Obukhov S P and Birk D E 1991 Phase transition and morphogenesis in a model biological system Phys. Rev. Lett. 67 2399-402

Forgacs G S and Newman A 1994 Phase transitions, interfaces, and morphogenesis in a network of protein fibers Int. Rev. Cytol. $150139-48$

Forgacs G, Newman S A, Polikova Z and Neumann A W 1994 Critical phenomena in model biological tissues Colloids Surf. B Biointerfaces 3 139-46

Foty R A, Forgacs G, Pfleger C M and Steinberg M S 1994 Liquid properties of embryonic tissues: measurement of interfacial tensions Phys. Rev. Lett. 72 2298-301

Foty R A, Pfleger C M, Forgacs G and Steinberg M S 1996 Surface tensions of embryonic tissues predict their mutual envelopment behavior Development 122 1611-20

Frenz D A, Akiyama S K, Paulsen D F and Newman S A 1989 Latex beads as probes of cell surface-extracellular matrix interactions during chondrogenesis: evidence for a role for amino-terminal heparin-binding domain of fibronectin Dev. Biol. 136 87-96

Fougnot C, Jozefowicz M and Rosenberg R D 1984 Catalysis of the generation of thrombin-antithrombin complex by insoluble anticoagulant polystyrene derivatives Biomaterials 5 94-9

Freyman T M, Yannas I V, Yokoo R and Gibson L J 2001 Fibroblast contraction of a collagen-GAG matrix Biomaterials 22 2883-91

Fung Y C 1993 Biomechanics: Mechanical Properties of Living Tissues (New York: Springer)

Gaill F, Lechaire J P and Denefle J P 1991 Fibrillar pattern of self-assembled and cell-assembled collagen: resemblance and analogy Biol. Cell 72 149-58
Gilbert S F 2003 Developmental Biology 7th edn (Sunderland, MA: Sinauer Associates)

Giraud-Guille M M 1996 Twisted liquid crystalline supramolecular arrangements in morphogenesis Int. Rev. Cytol. 166 59-101

Glazier J A and Graner F 1993 A simulation of the differential adhesion driven rearrangement of biological cells Phys. Rev. E $472128-54$

Godt D and Tepass U 1998 Drosophila oocyte localization is mediated by differential cadherin-based adhesion Nature 395 387-91

Gonzalez-Reyes A and St Johnston D 1998 The Drosophila AP axis is polarised by the cadherin-mediated positioning of the oocyte Development 125 3635-44

Gullberg D E and Lundgren-Akerlund E 2002 Collagen-binding I domain integrins-what do they do? Prog. Histochem. Cytochem. 37 3-54

Heintzelman K F, Phillips H M and Davis G S 1978 Liquid-tissue behavior and differential cohesiveness during chick limb budding J. Embryol. Exp. Morphol. 47 1-15

Hutson M S, Tokutake Y, Chang M-S, Bloor J W, Venakides S, Kiehart D P and Edwards G S 2003 Forces for morphogenesis investigated with laser microsurgery and quantitative modeling Science 300 145-9

Ingber D E 2003 Tensegrity: II. How structural networks influence cellular information processing networks J. Cell Sci. 116 1397-408

Jozefowicz M and Jozefonvicz J 1997 Randomness and biospecificity: random copolymers are capable of biospecific molecular recognition in living systems Biomaterials $\mathbf{1 8}$ 1633-44

Karim M A, Miller D D, Farrar M A, Eleftheriades B H, Reddy B H, Breland C M and Samarel A M 1995 Histomorphometric and biochemical correlates of arterial procollagen gene expression during vascular repair after experimental angioplasty Circulation 91 2049-57

Linsenmayer T F, Fitch J M, Gordon M K, Cai C X, Igoe F, Marchant J K and Birk D E 1998 Development and roles of collagenous matrices in the embryonic avian cornea Prog. Retin. Eye Res. 17 231-65

McPherson J M, Sawamura S J, Condell R A, Rhee W and Wallace D G 1988 The effects of heparin on the physicochemical properties of reconstituted collagen Coll. Relat. Res. 8 65-82

Miranti C K and Brugge J S 2002 Sensing the environment: a historical perspective on integrin signal transduction Nat. Cell Biol. 4 E83-E90

Müller O, Gaub H E, Barmann M and Sackmann E 1991 Viscoelastic moduli of sterically and chemically cross-linked actin networks in the dilute to semi-dilute regime: measurements by an oscillating disc rheometer Macromolecules 24 3110-20

Nakagawa S, Pawelek P and Grinnell F 1989 Extracellular matrix organization modulates fibroblast growth and growth factor responsiveness Exp. Cell Res. 182 572-82

Nakatsuji N, Snow M H and Wylie C C 1986 Cinemicrographic study of the cell movement in the primitive-streak-stage mouse embryo J. Embryol. Exp. Morphol. 96 99-109

Newgreen D F 1989 Physical influences on neural crest cell migration in avian embryos: contact guidance and spatial restriction Dev. Biol. 131 136-48

Newman S A 1998 Networks of extracellular fibers and the generation of morphogenetic forces Dynamical Networks in Physics and Biology ed D Beysens and G Forgacs (Berlin: Springer) pp 139-48

Newman S, Cloître M, Allain C, Forgacs G and Beysens D 1997 Viscosity and elasticity during collagen assembly in vitro: relevance to matrix-driven translocation Biopolymers 41 $337-47$

Newman S A and Comper W D 1990 'Generic' physical mechanisms of morphogenesis and pattern formation Development $1101-18$ 
Newman S A, Frenz D A, Hasegawa E and Akiyama S K 1987 Matrix-driven translocation: dependence on interaction of amino-terminal domain of fibronectin with heparin-like surface components of cells or particles Proc. Natl Acad. Sci. USA $\mathbf{8 4}$ $4791-5$

Newman S A, Frenz D A, Tomasek J J and Rabuzzi D D 1985 Matrix-driven translocation of cells and nonliving particles Science 228 885-9

Newman S A and Tomasek J J 1996 Morphogenesis of connective tissues Extracellular Matrix (Molecular Components and Interactions vol 2) ed W D Comper (Amsterdam: Harwood Academic) pp 335-369

Roy P, Petroll W M, Cavanagh H D and Jester J V 1999 Exertion of tractional force requires the coordinated up-regulation of cell contractility and adhesion Cell Motil. Cytoskeleton $\mathbf{4 3}$ 23-34

Sahimi M 1994 Applications of Percolation Theory (London: Taylor and Francis)

Shafrir Y and Forgacs G 2002 Mechanotransduction through the cytoskeleton Am. J. Physiol. Cell Physiol. 282 C479-C486

Shafrir Y, ben-Avraham D and Forgacs G 2000 Trafficking and signaling through the cytoskeleton: a specific mechanism J. Cell Sci. $1132747-57$

Sawhney R K and Howard J 2002 Slow local movements of collagen fibers by fibroblasts drive the rapid global self-organization of collagen gels J. Cell Biol. 157 1083-91
Stauffer D and Aharony A 1992 Introduction to Percolation Theory 2nd edn (London: Taylor and Francis)

Steinberg M S 1978 Specific cell ligands and the differential adhesion hypothesis: How do they fit together? Specificity of Embryological Interactions ed D R Garrod (London: Chapman and Hall) pp 97-130

Steinberg M S and Poole T J 1982 Liquid behavior of embryonic tissues Cell Behavior ed R Bellairs and A S G Curtis (Cambridge: Cambridge University Press) pp 583-607

Steinberg M S and Takeichi M 1994 Experimental specification of cell sorting, tissue spreading, and specific spatial patterning by quantitative differences in cadherin expression Proc. Natl Acad. Sci. USA 91 206-9

Tanaka M and Tickle C 2004 Tbx 18 and boundary formation in chick somite and wing development Dev. Biol. 268 470-80

Thorogood P V and Hinchliffe J R 1975 An analysis of the condensation process during chondrogenesis in the embyronic hind limb J. Embryol. Exp. Morphol. 33 581-606

Veis A 1996 Dentin Extracellular Matrix (Tissue Function vol 1) ed W D Comper (Amsterdam: Harwood Academic) pp 41-76

Zahalak G I, Wagenseil J E, Wakatsuki T and Elson E L 2000 A cell-based constitutive relation for bio-artificial tissues Biophys. J. 79 2369-81 